\title{
Upregulation of Vascular Endothelial Growth Factor Expression in the Kidney Could Be Reversed Following Treadmill Exercise Training in Type I Diabetic Rats
}

\author{
Muhammed Al-Jarrah ${ }^{\mathrm{a}, \mathrm{d}}$, Nour Erekat ${ }^{\mathrm{b}}$, Ahed Al Khatib ${ }^{\mathrm{c}}$
}

\begin{abstract}
Background: Nephropathy is a significant complication of diabetes mellitus, which is associated with high morbidity and mortality. Exercise training has been shown to have renoprotective effects in diabetes. Unregulated vascular endothelial growth factor (VEGF) has been demonstrated in the diabetic kidney. Thus, the aim of our study is to illustrate the impact of endurance exercise training on the renal VEGF expression in type I diabetic rats.
\end{abstract}

Methods: Forty normal Sprague-Dawley rats were randomly divided into the following equal groups: sedentary control (SC), exercised control (EC), sedentary diabetic (SD) rats and exercised diabetic (ED) rats. Then, diabetes mellitus was induced by streptozotocin in the rats in the two diabetic groups. The expression of VEGF in the renal tissue in each of the four different groups was assessed by immunohistochemistry.

Results: Renal VEGF expression was significantly $(\mathrm{P}<0.01)$ higher in SD compared with that in SC. However, exercise training significantly $(\mathrm{P}<0.01)$ reduced VEGF expression in the renal tissue in ED compared with that in SD.

Conclusion: Our present data suggest that treadmill exercise training suppressed diabetes-induced upregulation in the renal VEGF expression.

Manuscript accepted for publication March 26, 2014

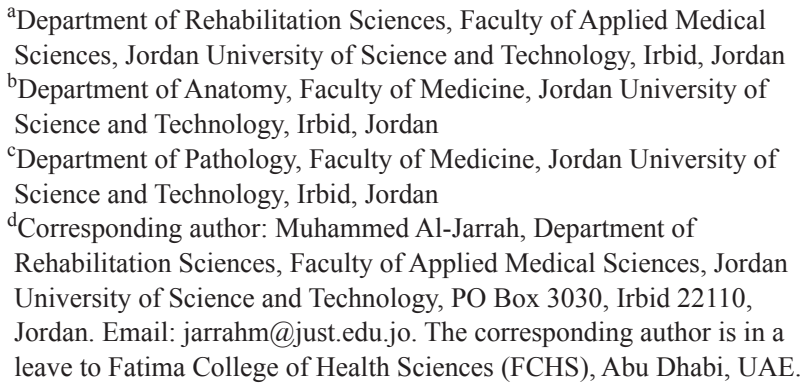

doi: http://dx.doi.org/10.14740/wjnu153e
Keywords: Type I diabetes; VEGF; Kidney; Treadmill exercise

\section{Introduction}

Nephropathy is a significant complication of diabetes mellitus [1]. Pathophysiology of diabetic nephropathy has been shown to involve the upregulation of VEGF expression [26]. VEGF is an angiogenic and permeability-inducing factor, whose altered expression has been demonstrated in many different tissues in diabetes mellitus [7-9]. For example, VEGF is increased in diabetic retinopathy and decreased in diabetic cardiac tissues [10-13].

VEGF has been implicated in the pathogenesis of many renal diseases, such as diabetic nephropathy, high proteininduced nephropathy and glomerulonephritis [2]. Diabetes mellitus is a major risk factor for nephropathy, which significantly increases the risk for morbidity and mortality in diabetic patients [14-20]. VEGF expression has been implicated in the pathogenesis of diabetic nephropathy [2-6]. VEGF has been upregulated in diabetic renal tissue [2-6]. In addition to that, the administration of antibodies against VEGF has improved early renal dysfunction in experimental type I diabetic rats and ameliorated long-term renal changes in obese type 2 diabetic mice [21, 22].

Exercise training has been shown to improve vascular endothelial function in patients with type I diabetes [23]. Treadmill exercise training has, indeed, suppressed VEGF upregulation in the diabetic retinas [24]. In addition to that, exercise training has improved the downregulated VEGF expression in the aging hearts, as well as in the skeletal muscle of diabetic patients $[25,26]$.

Regular moderate exercise has been reported to reduce advanced glycation and ameliorate early diabetic nephropathy in obese Zucker rats [27]. However, the impact of treadmill exercise training on VEGF expression has never been examined in diabetic renal tissue. Therefore, using immunohistochemistry and light microscopy, the goal of this study was to assess the effect of treadmill exercise training on VEGF expression in kidneys from rats with streptozotocininduced diabetes mellitus. 


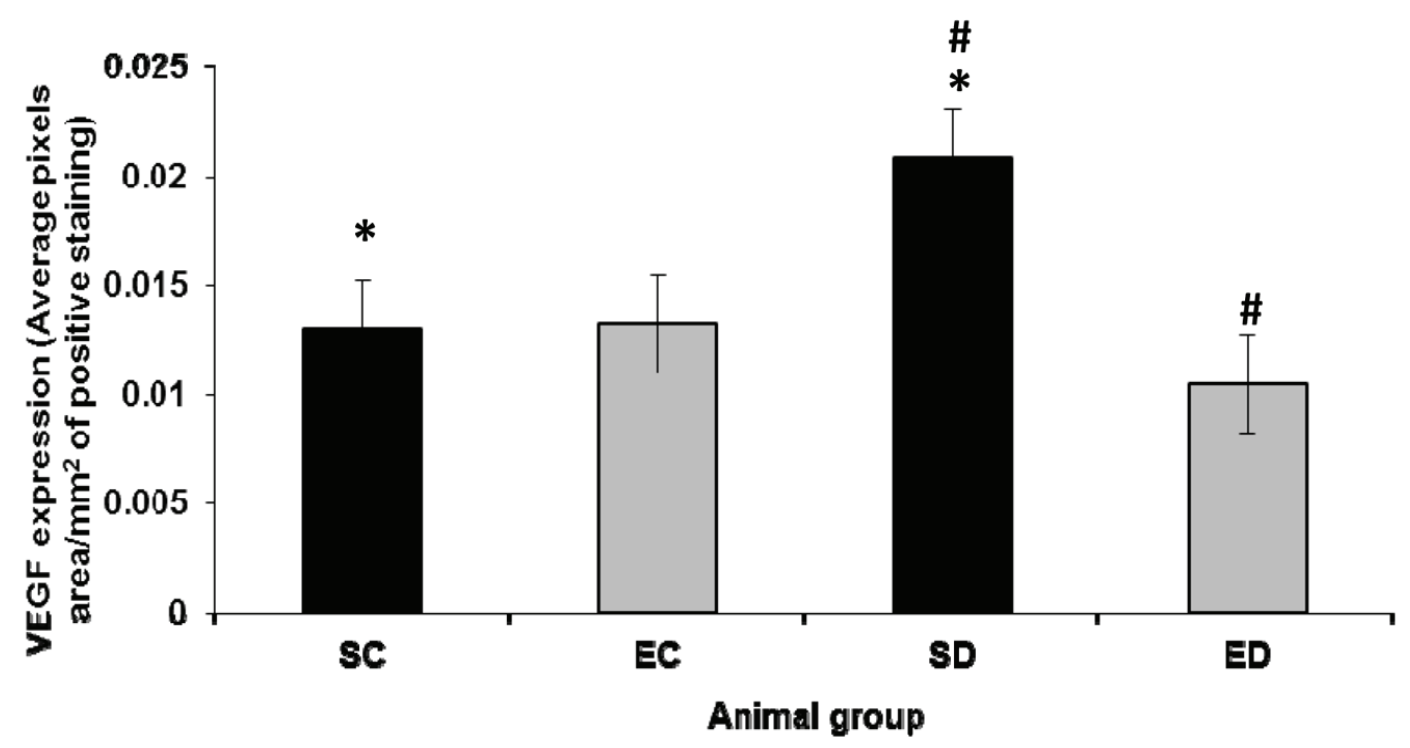

Figure 1. Expression of VEGF in the kidney. The expression level of VEGF increased significantly in the diabetic sedentary group compared to sedentary control groups $\left(P<0.01,{ }^{*}\right)$. Exercise training significantly decreased the expression level of VEGF $(P<0.01, \#)$ in diabetic rats. SC: sedentary control; EC: exercised control; SD: sed $\urcorner$ entary diabetic; ED: exercised diabetic.

\section{Materials and Methods}

\section{Animals}

Forty Sprague-Dawley rats were randomly divided into the four following groups: sedentary control $(\mathrm{SC}, \mathrm{n}=10)$, exercised control $(\mathrm{EC}, \mathrm{n}=10)$, sedentary diabetic $(\mathrm{SD}, \mathrm{n}=10)$ and exercised diabetic $(\mathrm{ED}, \mathrm{n}=10)$. Animals were housed in individual cages at $22 \pm 1{ }^{\circ} \mathrm{C}$ in a controlled room with a 12:12 light:dark cycle. The animals were allowed free access to standard chow and water. Animal care and experiments were performed in accordance with the research committee guidelines for animal experimentation at Jordan University of Science and Technology. Rats in the two diabetic groups were intraperitoneally injected with alloxan $(120 \mathrm{mg} / \mathrm{kg})$. Simultaneously, rats in the two control groups were intraperitoneally injected with saline $(120 \mathrm{mg} / \mathrm{kg})$. Three days later, fasting blood glucose above $250 \mathrm{mg} / \mathrm{dL}$ was detected in rats in the two diabetic groups confirming successful induction of diabetes.

\section{Exercise protocol}

The rats were exercised according to the exercise training protocol previously described and suggested to provide adequate systemic and cellular adaptations with this level of aerobic exercise [14]. Briefly, rats in the exercised control and exercised diabetic groups were running on a custom treadmill with eight separate lanes, at a speed of $18 \mathrm{~m} / \mathrm{min}$, $40 \mathrm{~min} /$ day for 5 days/week. In order to expose sedentary rats to the same environment as the exercised groups of animals, sedentary rats were transported daily to the training room simultaneously with the exercised rats.

\section{Immunohistochemistry of VEGF in the kidney}

After dissecting the kidneys, they were fixed in $4 \%$ paraformaldehyde. Then, $5 \mu \mathrm{m}$ thick paraffin-embedded sections were prepared and processed via immunohistochemistry according to the protocol described previously [15]. Briefly, the sections were deparaffinized in xylene for 2 min twice, and subsequently rehydrated through consecutively descending dilutions of alcohol $(100 \%, 90 \%, 80 \%$ and $70 \%)$ ending in water ( 2 min each step). After that, the sections were treated in the reveal solution (RV1000M; Biocare Medical, Concord, CA) in the Decloaking chamber (Biocare Medical) for $2 \mathrm{~min}$. Subsequently, $5 \mathrm{~min}$ incubation of the sections with $3 \%$ hydrogen peroxide in methanol was used to block endogenous peroxidase activity. After washing the sections in phosphate-buffered saline (PBS), they were incubated with anti-VEGF antibody (Santa Cruz Biotechnology, Santa Cruz, CA), diluted according to vendor instructions, for $1 \mathrm{~h}$ at room temperature, and then washed with PBS. Then, sections were incubated with biotinylated secondary antibody (LSAB kit, Dako Carpinteria, CA) for $15 \mathrm{~min}$ at room temperature, and subsequently washed with PBS. Sections were incubated with streptavidin horse radish peroxidase (LSAB kit, Dako) for 15 min at room temperature and washed with PBS. Finally, 3,3'-diaminobenzidine substrate was applied for 2 min or longer, until the desired color in- 
tensity was developed, and then the slides were washed with tap water to stop the reaction. Negative control slides were processed without the primary antibody. All sections were counterstained with hematoxylin and viewed under the light microscopy.

\section{Data collection and analysis}

The sections were photographed with digital camera. Ten slides from each animal of all 10 animals in each of the four groups were analyzed by counting the total pixels area occupied by positive staining, using Adobe Photoshop software, as described previously $[15,16]$. VEGF expression was analyzed, in the renal tissue from the different groups, and statistically compared among the four different groups using one-way ANOVA followed by independent samples t-test. Differences in VEGF expression were considered statistically significant at $\mathrm{P}$ value $<0.05$.

\section{Results}

Immunohistochemical staining revealed a similar distribution of VEGF expression in the kidneys from all groups, being localized in the epithelial glomerular cells and distal tubules present in the renal cortex. VEGF immunoreactivity has also been observed in the collecting ducts. VEGF expression was detected in both sedentary and exercised control kidneys. However, VEGF immunoreactivity was obviously increased in the diabetic kidney. On the other hand, exercise training conspicuously decreased VEGF immunoreactivity in the kidneys from exercised diabetic rats.

Streptozotocin-induced diabetes has statistically significantly increased VEGF expression in the kidney $(\mathrm{P}<$ 0.01) when compared with that in the control kidney (Fig. 1). However, treadmill exercise training has statistically insignificantly increased $(\mathrm{P}>0.05)$ renal VEGF expression in the control group (Fig. 1). On the other hand, renal VEGF expression is statistically significantly decreased $(\mathrm{P}<0.01)$ in the exercised diabetic group when compared with that in the sedentary diabetic group (Fig. 1).

\section{Discussion}

This is the first study to reveal the impact of treadmill exercise training on VEGF expression in the kidney of rats with streptozotocin-induced diabetes mellitus. Our analysis suggests that treadmill exercise training suppressed VEGF upregulation in the renal tissues of diabetic rats.

VEGF is normally expressed in the renal tissue, particularly in glomerular podocytes, distal tubules and collecting ducts $[28,29]$. This is consistent with our finding of VEGF detection in the kidneys of control rats (Fig. 1). VEGF has been suggested to play a role in the formation and maintenance of the glomerular capillary endothelial fenestrations $[30,31]$. In addition to that, VEGF has been considered as an important factor for nephrogenesis [32, 33]. Our results reveal a statistically insignificant increase in the VEGF expression in the renal tissues of the exercised controls compared with the non-exercised controls. Our findings are in agreement with the previous studies showing insignificant increase in VEGF expression in many tissues, such as skeletal muscles and heart, following endurance exercise training $[26,34,35]$. However, although VEGF protein expression significantly increased 1 day after exercise training in intact mice, it gradually returned to baseline after 4 days [36].

Our results reveal VEGF upregulation in the kidneys following the induction of diabetes mellitus by streptozotocin (Fig. 1). Incubating cultured podocytes in high concentrations of glucose have been shown to increase VEGF mRNA expression [37]. Thus, the increased VEGF levels in the diabetic kidney revealed by our results are consistent with the previous studies suggesting hyperglycemia-induced VEGF upregulation, which has been assumed to underlie some of the renal alterations causing the development of diabetic nephropathy $[3,38,39]$.

Exercise training has been suggested to have a beneficial effect on diabetic nephropathy [40]. To examine the mechanism by which exercise training may beneficially affect diabetic nephropathy, we tested the impact of treadmill exercise training on the renal VEGF expression in rats with streptozotocin-induced diabetes mellitus. Upregulation of VEGF expression has been demonstrated in retinas of diabetic patients and animals [41]. However, VEGF upregulation has been attenuated in diabetic retinas following endurance exercise training [24]. Similarly, our results (Fig. 1) reveal decreased renal VEGF levels in rats with streptozotocininduced diabetes mellitus following treadmill exercise training. The decreased levels of VEGF in the diabetic kidney after exercise training, shown by our study (Fig. 1), may explain the improved vascular endothelial function in diabetic patients following exercise training [23]. Regular moderate exercise has been suggested to reduce advanced glycation and ameliorate early diabetic nephropathy in obese Zucker rats [27]. Thus it can be concluded that exercise training reduces VEGF upregulation in the diabetic kidney by decreasing the hyperglycemia resulting from the improved glucose uptake due to the increased insulin sensitivity as suggested by previous reports [42-44].

In conclusion, the present study is the first to report the impact of endurance exercise training on VEGF expression in kidneys from rats with streptozotocin-induced diabetes mellitus. In summary, treadmill exercise training attenuates streptozotocin-induced diabetes mellitus-induced upregulation of VEGF expression in kidney. Thus VEGF inhibition might be considered as a therapeutic strategy in the treatment diabetic nephropathy. 


\section{Acknowledgement}

This study was financially supported by The Deanship of Research at Jordan University of Science and Technology, Irbid, Jordan.

\section{References}

1. Krolewski AS, Canessa M, Warram JH, Laffel LM, Christlieb AR, Knowler WC, Rand LI. Predisposition to hypertension and susceptibility to renal disease in insulin-dependent diabetes mellitus. N Engl J Med. 1988;318(3):140-145.

2. Schrijvers BF, Flyvbjerg A, De Vriese AS. The role of vascular endothelial growth factor (VEGF) in renal pathophysiology. Kidney Int. 2004;65(6):2003-2017.

3. Khamaisi M, Schrijvers BF, De Vriese AS, Raz I, Flyvbjerg A. The emerging role of VEGF in diabetic kidney disease. Nephrol Dial Transplant. 2003;18(8):14271430.

4. Nakagawa T, Kosugi T, Haneda M, Rivard CJ, Long DA. Abnormal angiogenesis in diabetic nephropathy. Diabetes. 2009;58(7):1471-1478.

5. Nakagawa T, Sato W, Kosugi T, Johnson RJ. Uncoupling of VEGF with endothelial NO as a potential mechanism for abnormal angiogenesis in the diabetic nephropathy. J Diabetes Res. 2013;2013:184539.

6. Cooper ME, Vranes D, Youssef S, Stacker SA, Cox AJ, Rizkalla B, Casley DJ, et al. Increased renal expression of vascular endothelial growth factor (VEGF) and its receptor VEGFR-2 in experimental diabetes. Diabetes. 1999;48(11):2229-2239.

7. Weis SM, Cheresh DA. Pathophysiological consequences of VEGF-induced vascular permeability. Nature. 2005;437(7058):497-504.

8. Aiello LP, Wong JS. Role of vascular endothelial growth factor in diabetic vascular complications. Kidney Int Suppl. 2000;77:S113-119.

9. Mahdy RA, Nada WM, Hadhoud KM, El-Tarhony SA. The role of vascular endothelial growth factor in the progression of diabetic vascular complications. Eye (Lond). 2010;24(10):1576-1584.

10. Boulton M, Foreman D, Williams G, McLeod D. VEGF localisation in diabetic retinopathy. Br J Ophthalmol. 1998;82(5):561-568.

11. Simo R, Hernandez C. Intravitreous anti-VEGF for diabetic retinopathy: hopes and fears for a new therapeutic strategy. Diabetologia. 2008;51(9):1574-1580.

12. Ray D, Mishra M, Ralph S, Read I, Davies R, Brenchley P. Association of the VEGF gene with proliferative diabetic retinopathy but not proteinuria in diabetes. Diabetes. 2004;53(3):861-864.

13. Farhangkhoee H, Khan ZA, Kaur H, Xin X, Chen S,
Chakrabarti S. Vascular endothelial dysfunction in diabetic cardiomyopathy: pathogenesis and potential treatment targets. Pharmacol Ther. 2006;111(2):384-399.

14. Ravid M, Brosh D, Ravid-Safran D, Levy Z, Rachmani R. Main risk factors for nephropathy in type 2 diabetes mellitus are plasma cholesterol levels, mean blood pressure, and hyperglycemia. Arch Intern Med. 1998;158(9):998-1004.

15. Viswanathan V, Tilak P, Kumpatla S. Risk factors associated with the development of overt nephropathy in type 2 diabetes patients: a 12 years observational study. Indian J Med Res. 2012;136(1):46-53.

16. Chiarelli F. Diabetic nephropathy-an evaluation of potential risk factors in young patients with type 1 diabetes mellitus. Nat Clin Pract Endocrinol Metab. 2008;4(6):318-319.

17. Molnar M, Wittmann I, Nagy J. Prevalence, course and risk factors of diabetic nephropathy in type-2 diabetes mellitus. Med Sci Monit. 2000;6(5):929-936.

18. Nielsen FS. Diabetic nephropathy in non-insulin dependent diabetes mellitus. Cardiovascular risk factors and antihypertensive treatment. Dan Med Bull. 2000;47(4):249-270.

19. Dobronravov VA. [Risk of chronic renal failure in patients with non-insulin dependent diabetes mellitus with diabetic nephropathy and the stage of permanent proteinuria and arterial hypertension: role of clinical factors]. Ter Arkh. 2000;72(6):52-54.

20. Rachmani R, Ravid M. Risk factors for nephropathy in type 2 diabetes mellitus. Compr Ther. 1999;25(6-7):366369.

21. Flyvbjerg A, Dagnaes-Hansen F, De Vriese AS, Schrijvers BF, Tilton RG, Rasch R. Amelioration of long-term renal changes in obese type 2 diabetic mice by a neutralizing vascular endothelial growth factor antibody. Diabetes. 2002;51(10):3090-3094.

22. de Vriese AS, Tilton RG, Elger M, Stephan CC, Kriz W, Lameire NH. Antibodies against vascular endothelial growth factor improve early renal dysfunction in experimental diabetes. J Am Soc Nephrol. 2001;12(5):9931000 .

23. Fuchsjager-Mayrl G, Pleiner J, Wiesinger GF, Sieder AE, Quittan M, Nuhr MJ, Francesconi C, et al. Exercise training improves vascular endothelial function in patients with type 1 diabetes. Diabetes Care. 2002;25(10):1795-1801.

24. Ji ES, Ko IG, Cho JW, Davis RW, Hwang GY, Jee YS, Lim BV. Treadmill exercise inhibits apoptotic neuronal cell death with suppressed vascular endothelial growth factor expression in the retinas of the diabetic rats. J Exerc Rehabil. 2013;9(3):348-353.

25. Iemitsu M, Maeda S, Jesmin S, Otsuki T, Miyauchi T. Exercise training improves aging-induced downregulation of VEGF angiogenic signaling cascade in hearts. 
Am J Physiol Heart Circ Physiol. 2006;291(3):H12901298.

26. Kivela R, Silvennoinen M, Touvra AM, Lehti TM, Kainulainen H, Vihko V. Effects of experimental type 1 diabetes and exercise training on angiogenic gene expression and capillarization in skeletal muscle. FASEB J. 2006;20(9):1570-1572.

27. Boor P, Celec P, Behuliak M, Grancic P, Kebis A, Kukan M, Pronayova N, et al. Regular moderate exercise reduces advanced glycation and ameliorates early diabetic nephropathy in obese Zucker rats. Metabolism. 2009;58(11):1669-1677.

28. Baderca F, Lighezan R, Dema A, Alexa A, Raica M. Immunohistochemical expression of VEGF in normal human renal parenchyma. Rom J Morphol Embryol. 2006;47(4):315-322.

29. Maharaj AS, Saint-Geniez M, Maldonado AE, D’Amore PA. Vascular endothelial growth factor localization in the adult. Am J Pathol. 2006;168(2):639-648.

30. Risau W. Development and differentiation of endothelium. Kidney Int Suppl. 1998;67:S3-6.

31. Satchell SC, Braet F. Glomerular endothelial cell fenestrations: an integral component of the glomerular filtration barrier. Am J Physiol Renal Physiol. 2009;296(5):F947-956.

32. Kitamoto Y, Tokunaga H, Tomita K. Vascular endothelial growth factor is an essential molecule for mouse kidney development: glomerulogenesis and nephrogenesis. J Clin Invest. 1997;99(10):2351-2357.

33. Tufro A, Norwood VF, Carey RM, Gomez RA. Vascular endothelial growth factor induces nephrogenesis and vasculogenesis. J Am Soc Nephrol. 1999;10(10):21252134.

34. Suzuki J. Microvascular angioadaptation after endurance training with L-arginine supplementation in rat heart and hindleg muscles. Exp Physiol. 2005;90(5):763-771.

35. Czarkowska-Paczek B, Zendzian-Piotrowska M, Bartlomiejczyk I, Przybylski J, Gorski J. Skeletal and heart muscle expression of PDGF-AA and VEGF-A after an acute bout of exercise and endurance training in rats.
Med Sci Monit. 2010;16(5):BR147-153.

36. Wu G, Rana JS, Wykrzykowska J, Du Z, Ke Q, Kang $\mathrm{P}$, Li J, et al. Exercise-induced expression of VEGF and salvation of myocardium in the early stage of myocardial infarction. Am J Physiol Heart Circ Physiol. 2009;296(2):H389-395.

37. Iglesias-de la Cruz MC, Ziyadeh FN, Isono M, Kouahou M, Han DC, Kalluri R, Mundel P, et al. Effects of high glucose and TGF-beta1 on the expression of collagen IV and vascular endothelial growth factor in mouse podocytes. Kidney Int. 2002;62(3):901-913.

38. Kanwar YS, Wada J, Sun L, Xie P, Wallner EI, Chen S, Chugh S, et al. Diabetic nephropathy: mechanisms of renal disease progression. Exp Biol Med (Maywood). 2008;233(1):4-11.

39. Kim BS, Chen J, Weinstein T, Noiri E, Goligorsky MS. VEGF expression in hypoxia and hyperglycemia: reciprocal effect on branching angiogenesis in epithelial-endothelial co-cultures. J Am Soc Nephrol. 2002;13(8):2027-2036.

40. Kurdak H, Sandikci S, Ergen N, Dogan A, Kurdak SS. The effects of regular aerobic exercise on renal functions in streptozotocin induced diabetic rats. J Sports Sci Med. 2010;9(2):294-299.

41. Hammes HP, Lin J, Bretzel RG, Brownlee M, Breier G. Upregulation of the vascular endothelial growth factor/ vascular endothelial growth factor receptor system in experimental background diabetic retinopathy of the rat. Diabetes. 1998;47(3):401-406.

42. Holten MK, Zacho M, Gaster M, Juel C, Wojtaszewski JF, Dela F. Strength training increases insulin-mediated glucose uptake, GLUT4 content, and insulin signaling in skeletal muscle in patients with type 2 diabetes. Diabetes. 2004;53(2):294-305.

43. Hansen PA, Nolte LA, Chen MM, Holloszy JO. Increased GLUT-4 translocation mediates enhanced insulin sensitivity of muscle glucose transport after exercise. J Appl Physiol (1985). 1998;85(4):1218-1222.

44. Goodyear LJ, Kahn BB. Exercise, glucose transport, and insulin sensitivity. Annu Rev Med. 1998;49:235-261. 